\title{
Jahreswarven im Sennesander?
}

\author{
Von Adolf Depre und Fritz Fezer \\ Mit 4 Abbildungen im Text
}

$\mathrm{Z}$ us ammenfassung: Die Turonkalke im südwestlichen Teutoburger Wald tragen auffallend mächtigen, splittrigen Schutt. Durch einige Aufschlüsse im Vorland, der "Senne", konnte dieses Material einige $\mathrm{km}$ verfolgt werden. Die Splitter werden dabei kleiner, etwas kantengerundet und immer stärker von glazio-fluviatilen Sanden unterbrochen. Der ziemlich regelmäßige Wechsei von Sand und Splittern könnte in einem jahreszeitlichen Wechsel von Solifluktion und Eisschmelze seine Ursache haben.

S u m mary: The western edge of the Teutoburger Wald near Bielefeld consists of Turon limestones. They are covered by thick layers of weathered lime plates. We found the same material in the plain "Senne", where the plates become smaller, loose their edges and are interrupted by sand layers. We suppose the glacier rivers transporting plates out of the warm Turon edge in spring and sand out of the Inlandeis in summer.

In den Jahren 1889 bis 1958 hat die Stadt Bielefeld und die Firma Windel in der S e n n e südwestlich des Teutoburger Waldes über $300 \mathrm{Mal}$ nach Trinkwasser gebohrt. Dabei stieß man in den vorgeschütteten Sanden, die beim Herannahen des Inlandeises abgelagert wurden, wie auch bei den nachgeschütteten, die erst beim Abschmelzen, daher über dem Geschiebemergel aufgeschüttet wurden, häufig auf „Sand mit Plänerkies“ (Mestwerdt, 1926 u. Mitt. d. Wasserwerks Bielefeld).

Seit einigen Jahren wird dieser Sander in großem Maßstab abgebaut: zum Aufschütten des Autobahndamms, für die Fabrikation von Kalksandsteinen und zum Straßenunterbau. Dadurch kann man sich von der Lagerung und Verbreitung ein besseres Bild machen: Der Sand wird immer von dünnen Bänkchen unterbrochen, die aus bis zu $5 \mathrm{~cm}$ langen, schwach kantengerundeten Kreidekalksplittern, vereinzelten rosa Kalken, wenigen Graniten $(1 \% \%)$ und viel Sand bestehen. Die Bänkchen werden nach unten häufiger und mächtiger, schließlich nehmen sie so zu, daß sich die Sandgewinnung nicht mehr lohnt. Die Kalksplitter liegen annähernd waagrecht (Abb. 3 u. 4). Vor der Mündung eines Quertals in die Senne werden diese Kalksplitterbänke seltener, vor den Turonkalkkämmen häufiger (Abb. 2). Nach Südwesten nimmt ihr Anteil im Sand rasch ab. So findet man in der $4 \mathrm{~km}$ vom Gebirge entfernten Sandgrube Schlingermann kaum noch Kreidekalke, sondern höchstens Sandstein- und Flammenmergelsplitter.

Süd

Nord

Höhe 240

Schutt $0,8 \mathrm{~m}$ machtig
Längstal

Schutt $3 \mathrm{~m}$ mächtig

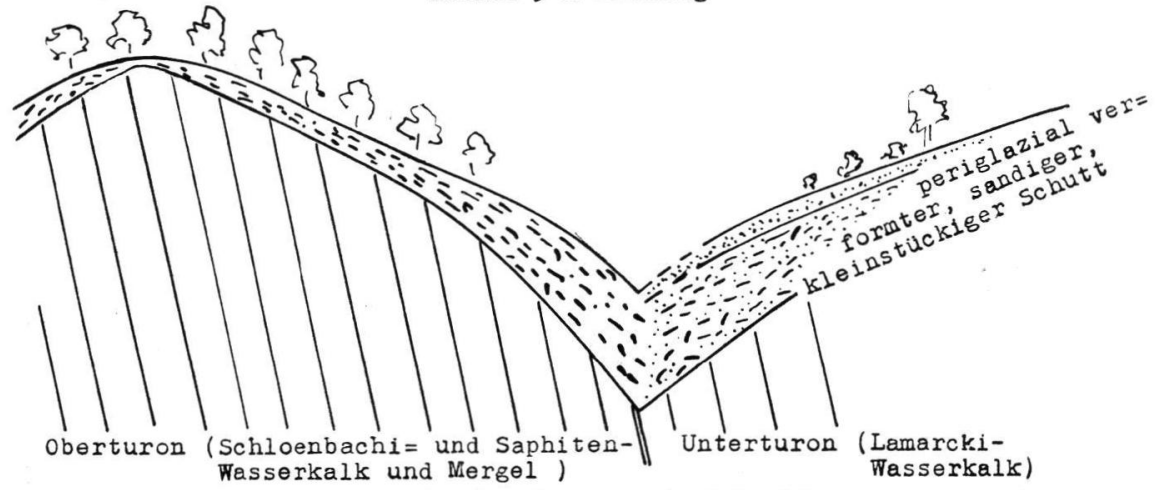

Abb. 1. Steinbruch Imkamp (I) in Spiegelsberge. 


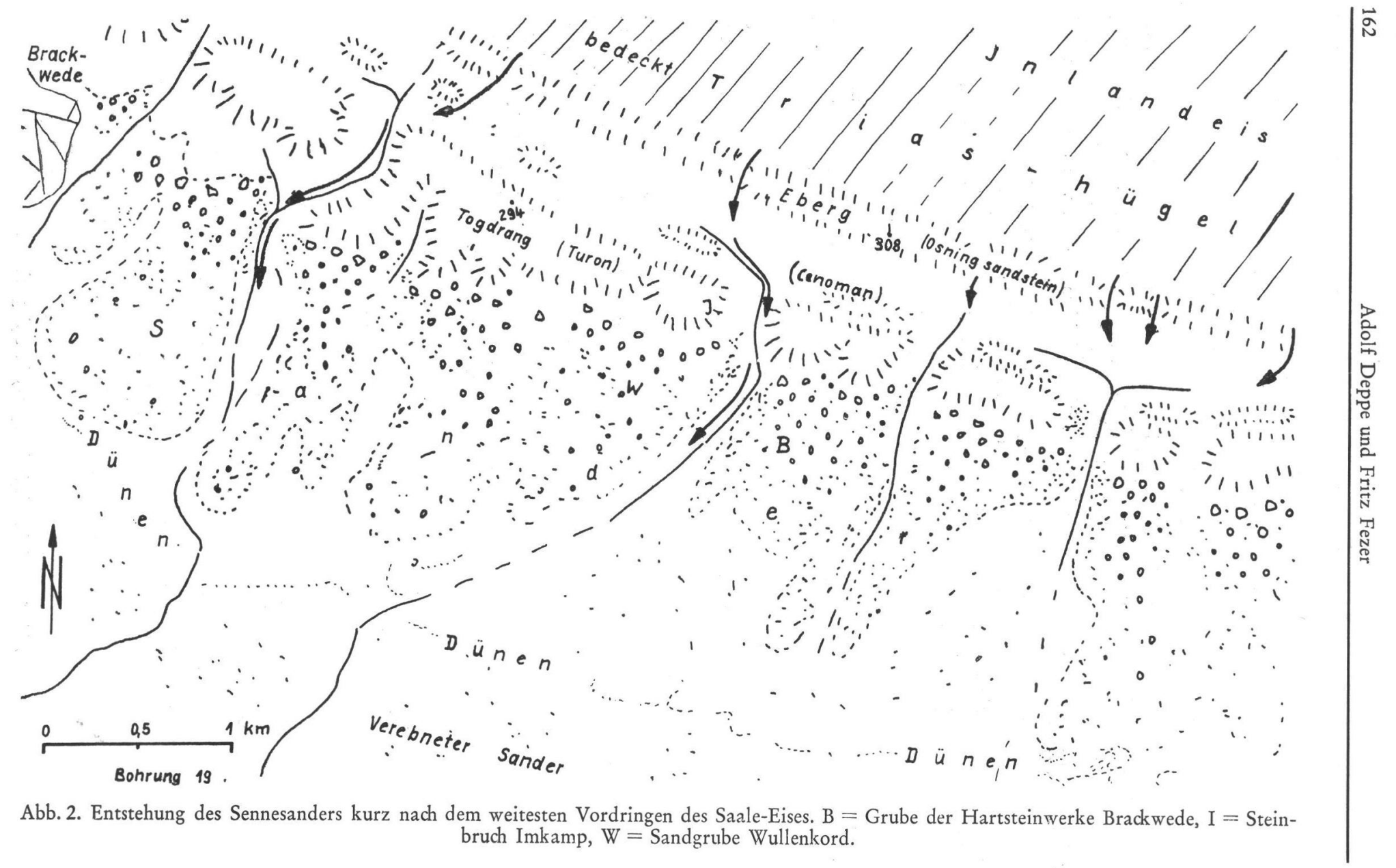




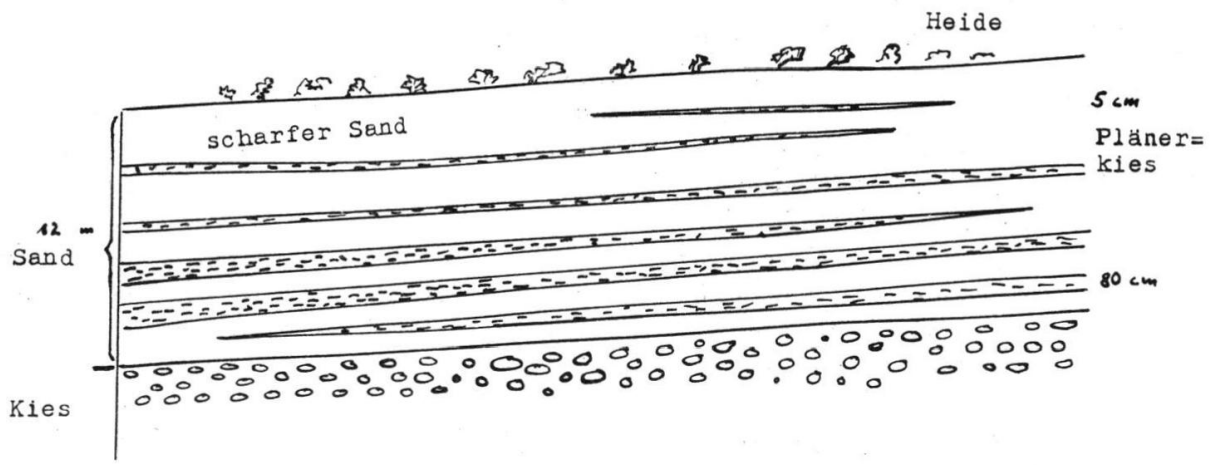

Abb. 3. Sandgrube Wullenkord (W) beim Sennefriedhof Brackwede.

Letztes Jahr konnte nun auch der Entstehungsraum der Kalksplitter beobachtet werden. Im Steinbruch Imkamp in Spiegelsberge (Abb. 1 und I in Abb. 2), also an einem Turonkalkkamm, war eine große Fläche freigelegt und die Deckschichten auf langer Front angeschnitten. In dem kleinen Längstälchen, das $100 \mathrm{~m}$ weiter unten in ein Quertal mündet, lag auf dem südlichen Hang ein lehmiger Kalkschutt mit bis zu $20 \mathrm{~cm}$ langen, scharfen Platten; auf der Nordseite enthielt er auch Sand. Außerdem war er dort von einem kalksteinfreien, lehmigen Sand bedeckt, der tropfen- oder keilförmig in den Schutt hinunter reichte. Wahrscheinlich ist der Schutt periglazial verformt und überhaupt durch Frost entstanden. Er ist hier viermal so mächtig wie auf gleicher Neigung und ähnlichem Gestein in anderen Mittelgebirgen, weil hier der Eisrand in der Nähe lag und weil der mergelige Kalk hier senkrecht steht und aus den Schichtfugen ständig Wasser aus der Tiefe gesogen werden konnte, während eine Frostverwitterung von waagrecht lagernden Mergelkalken bald zum Stillstand käne.

Man findet also im Gebirge selbst groben, lehmigen Kalkschutt, im nahen Vorland feinstückige Kalkschuttbänke im Sand und in größerer Entfernung vom Gebirge noch

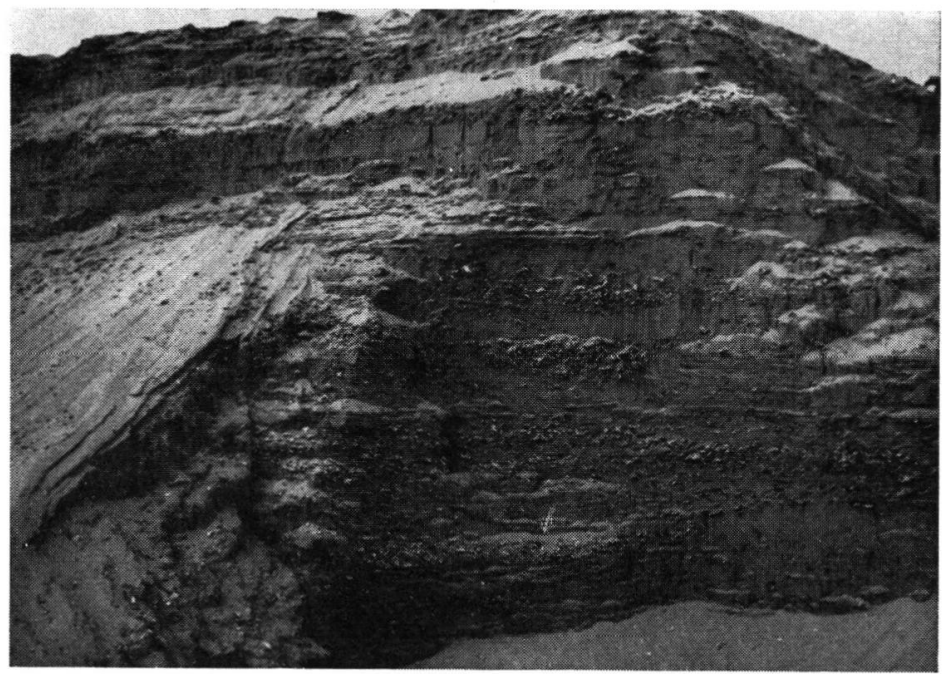

Abb. 4. Sandgrube Wullenkord beim Sennefriedhof Brackwede. Die Bänke von Kalkschutt („Plänerkies") werden nach unten zahlreicher und mächtiger. - Aufn. Dr. H. PAPE. 
einzelne Splitter. In der Art, Zurundung und Lagerung sind sie sich aber so ähnlich, daß wir alle in einem Zusammenhang sehen mïssen:

Der Schutt floß in den Längstälern aus dem Gebirge ab, mündete in den Quertälern ins Schmelzwasserbett des Saale-Eises oder auch direkt auf den Vorlandsander und wurde vom Wasser noch weit verfrachtet. In der Sandgrube Wullenkord (W), in den Gruben der Hartsteinwerke Brackwede (B, westl. u. östl. d. Straße Buschkamp-Gr. Bockermann) und Oerlinghausen (östl. d. Straße Oerlinghausen-Schloß Holte) ist Sand und Kalkschutt in ziemlich regelmäßigem Wechsel gelagert. Man kann sich das so erklären, daß den Schmelzwasserbächen in der einen Jahreszeit mehr Sand, in der anderen mehr Kreideschutt zufloß. Es könnte sein, daß einige Zeit nach dem Saale-Maximum sich das Eis am Nordrand des Teutoburger Waldes staute, im Sommer um einige Meter abschmolz und die Schmelzwässer in den Quertälern (Pfeile in Abb. 2) nicht mehr über die Pässe gelangen konnten. Die Verfasser halten es aber für wahrscheinlicher, daß der Frostschutt auf den so besonders warmen Südwesthängen schon im Frühjahr auftaute und ins Fließen kam, während das Inlandeis erst einige Wochen später kräftig abschmolz. Die Wechsellagerung ist jedoch längst nicht so regelmäßig wie in den Bändertonen; man kann daher nicht die Schichten auszählen, um die Ablagerungszeit in Jahren zu ermitteln, wie es De GeER getan hat.

\section{Literatur:}

Mestwerdt, A.: Erläuterungen zu Blatt Brackwede, 1926.

Franken, A.: Bodenstrukturen am Südhang des Teutoburger Waldes. - Jahresber. d. naturw. Ver. Bielefeld, 1952.

Manuskript eingeg. 20. 8. 1959.

Anschrift der Verf.: A. Deppe, Bielefeld, Sebastian-Bach-Straße 7 und Dr. Fritz Fezer, HeilbronnBöckingen, Heidenrain 13. 